EPJ Web of Conferences 80,00018 (2014)

DOI: $10.1051 /$ epj conf/ 20148000018

(C) Owned by the authors, published by EDP Sciences, 2014

\title{
Selected Issues in Non-Perturbative QCD
}

\author{
Luca Trentadue $1,2, a$ \\ ${ }^{1}$ Dipartimento di Fisica e Scienze della Terra, Università di Parma, Parma, Italy \\ ${ }^{2}$ INFN Sezione di Milano Bicocca, Milano, Italy
}

\begin{abstract}
A short selection of the topics presented and discussed at the Workshop on the long term strategy of INFN-CSN1: The next 10 Years of accelerator based esperiments. Isola d'Elba, May 21-24, 2014 - Working group " NP-QCD"
\end{abstract}

\section{Introduction}

From May 22nd-24th 2014, as an integral part of the INFN "What's Next" process started with the kick-off meeting held in Rome on April 7-8, 2014, a workshop organized by the INFN-CSN1 (Commissione Scientifica Nazionale I - National Scientific Committee I) has been held at "La Biodola" in the Isola d'Elba [1]. The aim of this workshop was "The development of a strategic plan for the CSN1 to be followed over the next 10-year timescale, in the context of a 20-year global vision for the field". The plan is "to identify clear scientific priorities, while taking into account technical readiness and financial feasibility of the potential new activities and infrastructures. The unique aspects of the science achievable with accelerator based experiments relative to other approaches also has to be emphasized".

Here we present a short and incomplete list of the topics that have been presented during the meetings of one of the four working groups: the Non Perturbative QCD Working Group ${ }^{1}$. A complete collection of the transparencies of the talks given in the parallel and plenary sessions can be found at the workshop site [1].

\section{Nucleon 3D Structure}

\subsection{Nucleon 3D Structure - Theory}

Presented by Marco Radici. The structure of the nucleons. The most relevant aspects. A key role is the kinematics of the infinite momentum frame (IMF) within the Deep Inelastic Framework and the collinear limit. Proposal of a new way of seeing already known facts: as the proton spin issue (see Fig.1) and the tomography of the nucleon (see Fig. 2). An extensive discussion of the Generalized Parton Distributions (GPD) functions, their model dependent parametrization and the connection with the lattice with an extensive list of references (for details see the transparencies on [1]). A discussion of the transverse momentum distribution (TMD) functions and the relevant role played by the soft gluon dynamics and the multiple gluon radiation resummation. As future directions the $\mathrm{LHeC}$ collider and the opening a new era of $3 \mathrm{D}$ view of the nucleons is envisaged. A comprehensive presentation about all the future $\mathrm{LHeC}$ projects has been presented by R. Ent [1].

\footnotetext{
ae-mail: luca.trentadue@unipr.it,luca.trentadue@cern.ch

${ }^{1}$ Convened by M. Anselmino, M. Ruspa and L. Trentadue.
} 


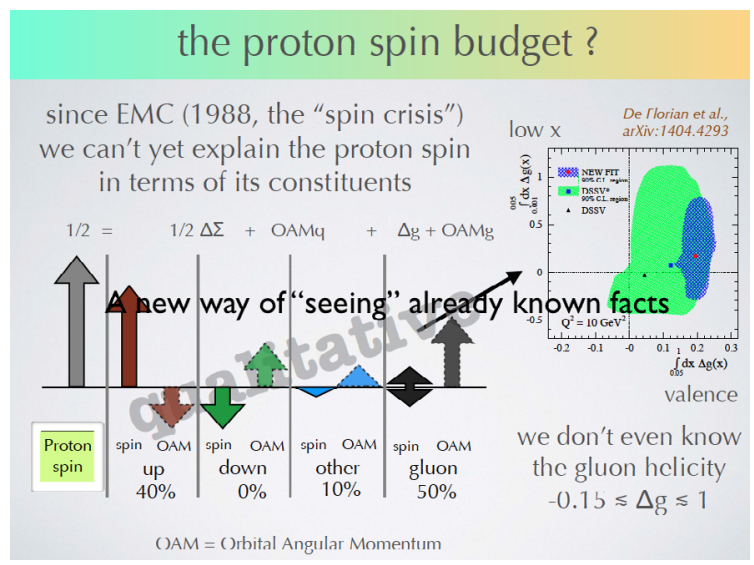

Figure 1. M. Radici. The list of the various contributions to the proton spin.

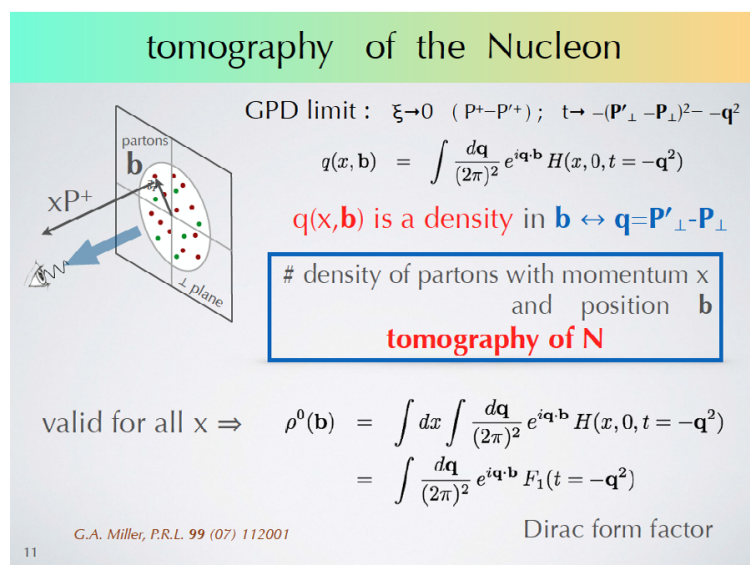

Figure 2. M. Radici. A pictorial view. The tomography of the Nucleon.

\subsection{Nucleon 3D Structure - Experimental data and perspectives}

Presented by A. Bressan. The extensive amount of the data obtained by the COMPASS experiment starting form the 2002 until 2014 with both polarized and unpolarized targets. The transversity measurement. The access to GPD from COMPASS in the future as well as the polarized Drell-Yan and the semi-inclusive Deep Inelastic Scattering for the TMD functions. The future of COMPASS (see Fig. 3 and Fig. 4).

\subsection{Drell-Yan Scattering and the structure of hadrons}

O. Denisov. Drell-Yan and Deep Inelastic Scattering can allow the access to the same physics quantities via different probes. Only if TMD function manifest themselves similarly one can claim the hadron structure is showing up. A list of FermiLab experiments and the proposal of DY@COMPASS (see Fig. 5). 


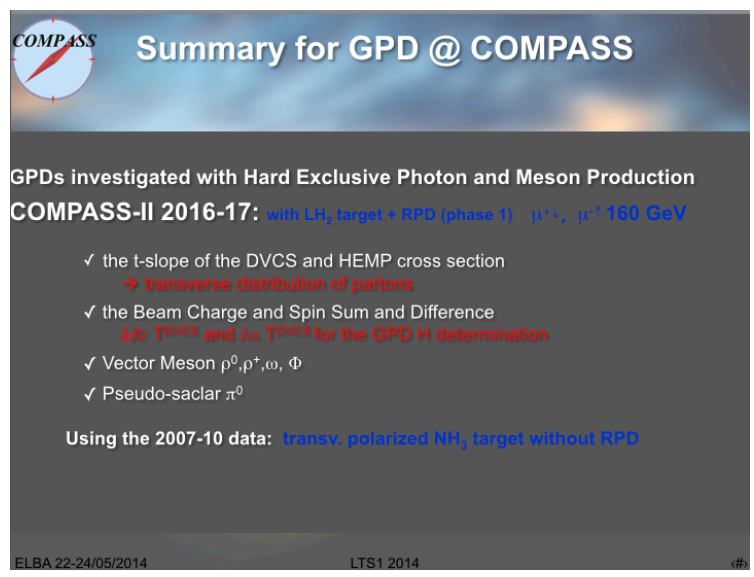

Figure 3. A. Bressan. GPD at COMPASS.

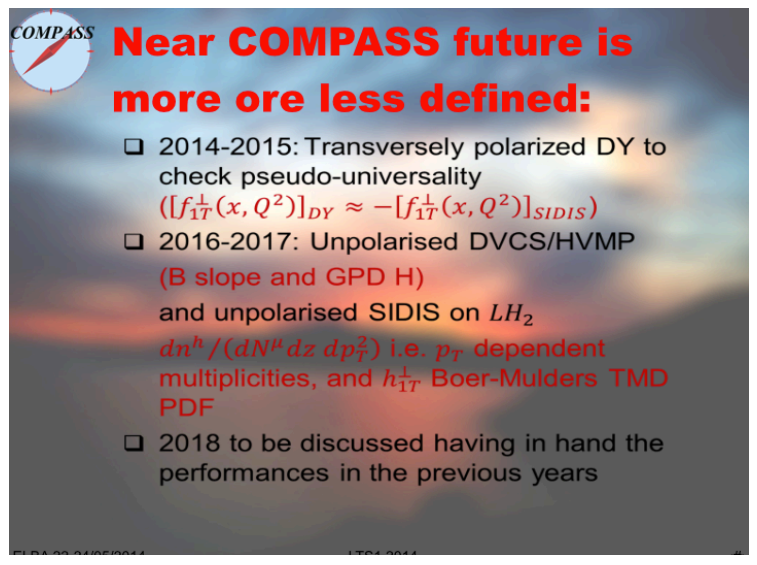

Figure 4. A. Bressan. COMPASS near future.

\subsection{Parton Distribution Function}

Presented by K. Wichmann. The crucial role played now and in the future LHC physics by the exact determination of the gluon structure function. A crucial issue (see Fig. 6).

\section{Multiple Parton Interaction}

Presented by L. Fanò. Multiple Parton Interactions (MPI) have been introduced to solve the unitarity problem generated by the fast raise of the inclusive hard pp cross sections at small $\mathrm{x}$. The approach turns out to be highly predictive on hadronic final states. MPI helps to probe proton matter distribution; understanding the collision dynamics and to define, at the best, backgrounds to new physics search. Several indications of MPI in pp collision can be shown. For the double parton interaction see Fig. 7. 


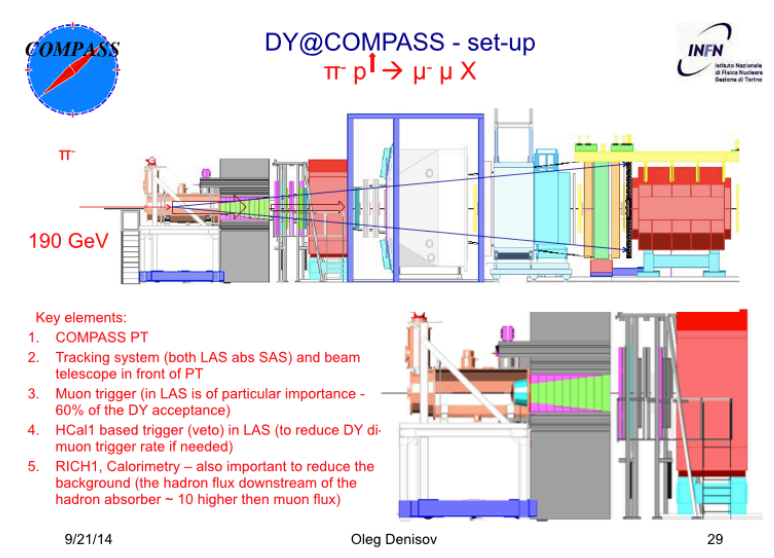

Figure 5. O. Denisov. DY@COMPASS.

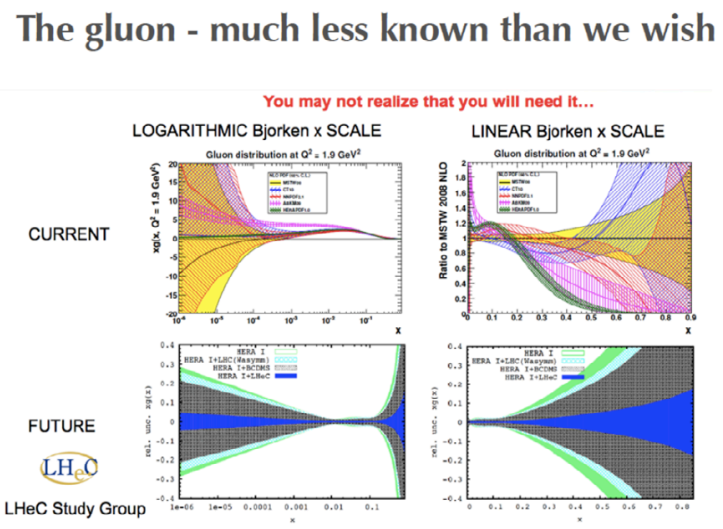

Figure 6. K. Wichmann. The indetermination on the gluon structure function.

\section{Cosmic ray physics at accelerators}

Presented by G. Mitsuka. The cosmic ray interactions can be reproduced at the accelerators (see Fig. 8). The analysis of data performed by the experiment LHCf at LHC has considered ElectroMagnetic showers events at the beginning, and has extended it after to the neutron events and to $\mathrm{p}-\mathrm{Pb}$ events. The analysis is still continuing.

\section{Spectroscopy}

Presented by R. Mussa. Striking regularities among the ground state mesons despite the large scale variation of the masses of the constituent quarks: s, c, b (see Fig. 9).

Related to spectroscopy is the project presented by Elena Santopinto of constituting a group of study on hadron spectroscopy and hybrids, a collaboration between experimentalist and theoreticians (see [1]). 


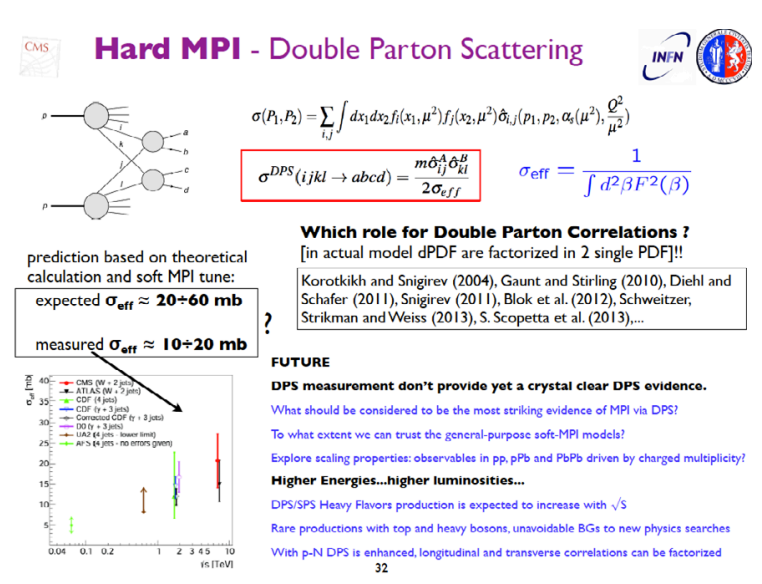

Figure 7. L. Fanò. The double parton scattering case.

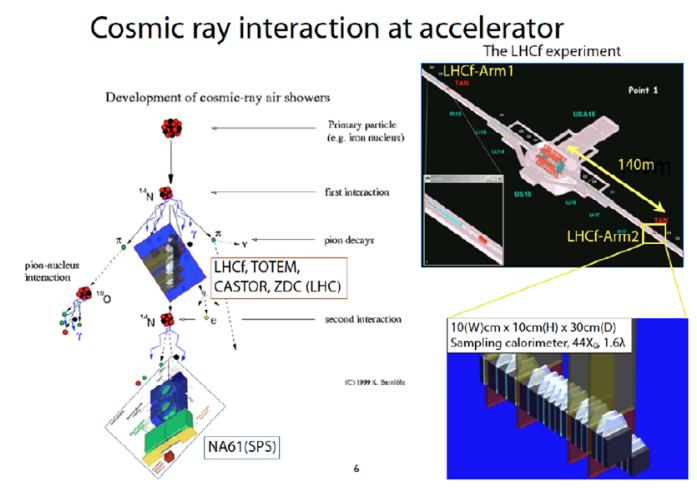

Figure 8. G. Mitsuka. Reproducing cosmic rays at the LHC.

\section{Diffraction}

\subsection{Theoretical aspects of diffraction}

Presented by F. Ceccopieri. Hard Diffraction in DIS has a complete perturbative QCD description, factorization theorems and parton evolution. At hadron colliders factorization cannot be proven apart from the case of inclusive Drell-Yan. When factorization fails it opens a new view on the Non Perturbative physics and on the hadronic structure. An extensive set of measures on hard and soft diffractions are ongoing at LHC. A very interesting channel is the diffractive Drell-Yan (Fig. 10) and a set of new open questions (Fig. 11). 


\section{EPJ Web of Conferences}

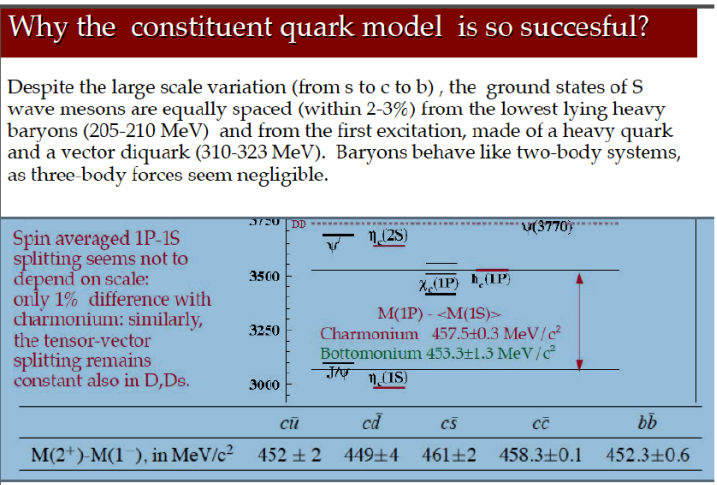

Figure 9. R. Mussa. Spin averaged 1P-1S splitting for s,c and b mesons.

\section{Prototype process: diffractive DY}

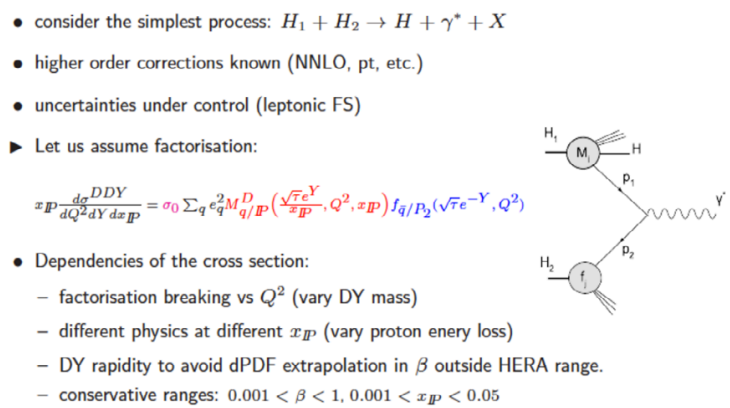

Figure 10. F. Ceccopieri. Diffractive Drell-Yan.

\section{Open questions}

- Can we correct the factorisation formula by a factor $S$ ?

$d \sigma \propto f^{D} \otimes f \otimes d \hat{\sigma} \otimes S(.$.

- which are the dependences of $S$ ?

- do we see the same partonic structure oberved at HERA?

- can be the cross section factorised at all?

- Compare Single and Double Diffraction $d \sigma \propto f^{D} \otimes f^{D} \otimes d \hat{\sigma} \otimes S^{\prime}(.$.

- what are the relations between $S$ and $S^{\prime}$ ?

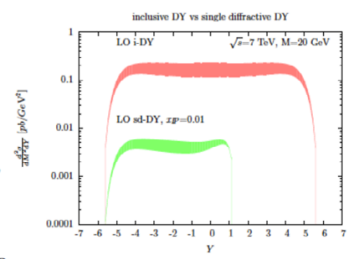

-What if one measures forward neutron instead of protons?

Figure 11. F. Ceccopieri. Open questions. 

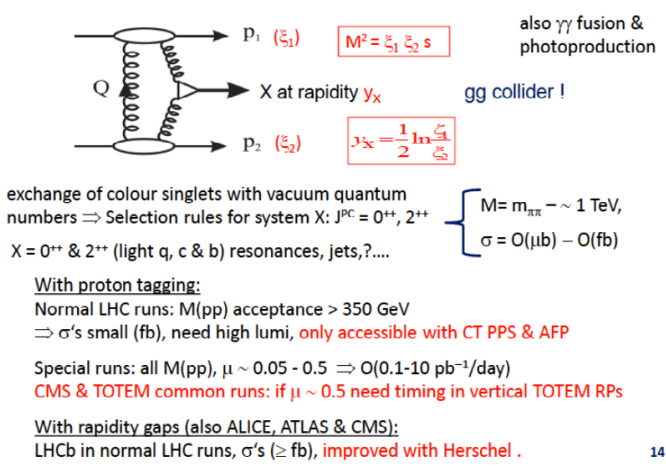

Figure 12. K. Osterberg. CEP processes.

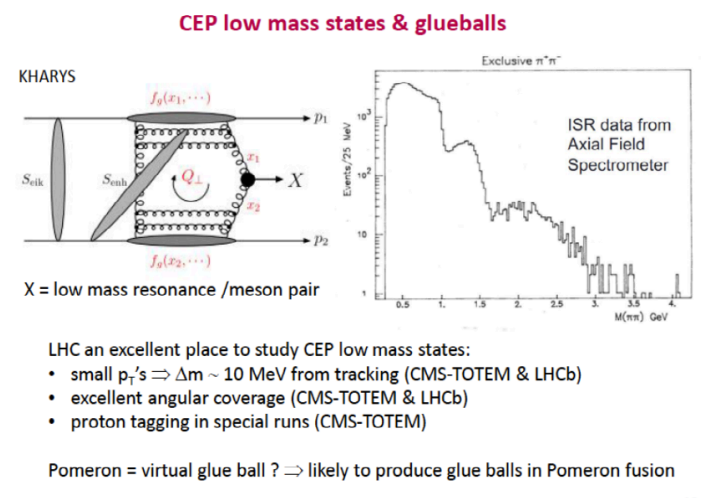

Figure 13. K. Osterberg. CEP processes - glueball production.

\subsection{Total, elastic and diffractive cross sections}

Presented by K. Osterberg. A new rich and very promising tool to investigate diffraction is given by the Central Exclusive production (CEP) (Fig. 12). It allows an extensive range of production processes from jets to low mass states and glueballs: a gluon collider with a known initial state energy (Fig. 13) .

\section{Highlights on confinement}

Presented by M. D'Elia. Understanding the Non Perturbative properties of QCD is amajor challenge. Confinement but deconfinement also: is the strongly interacting matter confined forever? (see Fig. 14). Some considerations (Fig.15) and some conclusions: goals and perspectives (Fig. 16). 


\section{EPJ Web of Conferences}

Deconfinement as a probe for Confinement

Is strongly interacting matter confined forever?

N. Cabibbo and G. Parisi (1975): a new, deconfined state of matter, corresponding to quark liberation, may exist in extreme conditions of high temperature or high baryon density.

The physics of the early Universe and of compact astrophysical objects may be described by states of matter completely away from our common experience.

Understanding how quarks and gluon deconfine, and what is the nature of the deconfined phase, may give us insight into confinement itself.

Figure 14. M. D’Elia. Deconfinement.

\section{Some considerations}

How can confinement be an absolute property of the QCD vacuum, and deconfining be just a smooth change of properties (no transition)?

Maybe one should understand what the deconfined thermal medium really is.

Experimental input (heavy ion): liquid like behavior (elliptic flow) and jet quenching.

In which sense a quark is deconfined, and what are its transport properties through the deconfining thermal medium?

Unfortunately, lattice $Q C D$ is ideally suited only for the study of equilibrium properties When considering real time dynamics, e.g. for transport properties, reaching a complete control over systematics is a very hard conceptual and numerical task.

(see M. Panero, K Rummukainen and A. Schaefer, PRL 112, 162001 (2014) for a recent study of soft mode contributions to jet quenching.)

Figure 15. M. D’Elia. Some considerations.

Conclusions: goals and perspectives

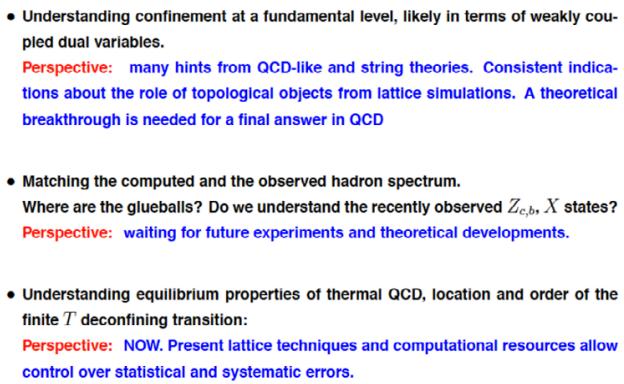

Figure 16. M.D’Elia. Conclusions. 
Let me make a final a comment. By trying to translate in the Italian language the words "New Physics" one may do it in two different ways: one way is to translate it as "Nuova Fisica", meaning new and unexpected phenomena or facts; a second one is "Fisica Nuova", meaning a new way of seeing and describing already known phenomena. We believe both should be considered as equally important.

\section{Acknowledgements}

I would like to thank all the colleagues who gave the presentations and contributed to the discussions, especially those who came from abroad. I am also very much grateful to Fulvia De Fazio and to Pietro Colangelo for inviting me at this lively Workshop.

\section{References}

[1] A complete description of the workshop contents and the entire collection of the transparencies can be found at the workshop web page: https://agenda.infn.it/conferenceDisplay.py?confId=7567 
